\title{
Probing the birth of super star clusters: Implications for massive star formation
}

\author{
Kelsey E. Johnson ${ }^{1,2}$ \\ ${ }^{1}$ Department of Astronomy, University of Virginia,Charlottesville, VA, 22903 USA \\ ${ }^{2}$ Hubble Fellow \\ email: kej7a@virginia.edu
}

\begin{abstract}
Super Star Clusters are one of the most extreme star forming environments in the universe, and the most massive and dense of these may be proto globular clusters. Like individual massive stars, the earliest stages of super star cluster evolution are deeply obscured, and therefore our knowledge about their birth environments is currently very incomplete. However, the study of natal super star clusters has become somewhat of a cottage industry in recent years, and the sample of such objects has been growing rapidly with high-quality long-wavelength data now available from a number of observatories. The natal super star clusters identified in thermalinfrared and radio observations represent the youngest stage of massive star cluster evolution yet observed. Their properties appear to be similar to those of ultracompact Hil regions in the Milky Way, but scaled up in total mass and luminosity. I will overview what we think we know about these objects based on existing observations, discuss their relationship to ultracompact HiI regions, present new models of their spectral energy distributions based on 3-D simulations, and outline some of the most significant gaps in our current understanding.
\end{abstract}

Keywords. stars: formation, Hit Regions, extinction, galaxies: star clusters, galaxies: starburst

\section{Introduction}

We know that nearly all massive stars form in clusters (see, e.g. de Wit, these proceedings). Therefore, if we wish to understand massive star formation in general, we must understand the clustered mode of star formation. Super star clusters (SSCs) are the most extreme type of stellar cluster (and also one of the most extreme star forming environ-

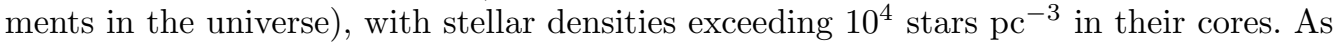
"extreme" objects, one might be tempted to simply think of them as cosmic curiosities. However, these objects are not only fascinating because of their extreme nature, but also because they hold clues to an important mode of star formation during the time of galaxy assembly. Specifically, most present-day research strongly supports the idea that SSCs are the adolescent precursors to the ancient globular clusters that are ubiquitous around massive galaxies in the local universe today. However, one should also bear in mind that the infant mortality rate of SSCs in likely to be quite high (possibly as high as 99\%, Fall \& Zhang 2001), and only the most robust of these objects will have survived to the ripe old age of present-day globular clusters. The low survival rate of SSCs suggests that they must have been formed prodigiously in the early universe, making this mode of star formation even more important for understanding the early evolution of of today's massive galaxies.

SSCs are also important components of galaxies in the local universe. These clusters can have an incredible impact on their surrounding interstellar medium (ISM), and in some cases even the intergalactic medium (IGM). Each of these clusters can host up to thousands of massive stars that have powerful stellar winds, and these stars will all die 
in a violent way within a few million years of each other. The collective effect of these massive stars (both in their lives and deaths!) can cause tremendous outflows, ionize large volumes of the ISM and IGM, and enrich vast amounts of interstellar material (e.g. Johnson et al. 2000, Heckman 2001, Martin, Kobulnicky, \& Heckman 2002)

Moreover, SSCs are also important tools for probing the universe. They are the most luminous type of "simple stellar population" (the constituent stars share the same age and metallicity), which makes them very useful "test particles" for investigating local galaxy properties. In addition, SSCs will be among the brightest point sources visible in galaxies out to large distances, which makes them very attractive targets for observations that must worry about surface brightness issues.

\section{The Foundations of Super Star Cluster Research}

Research on SSCs is rapidly approaching a crossroads, and it is therefore worth briefly reviewing the tremendous advances that have been made in this field over roughly the last decade. Perhaps most importantly, we have learned that globular cluster formation continues today. This discovery came to the forefront of cluster research with the Hubble Space Telescope, and caused a major revision of the prevailing theories of globular cluster formation and galaxy evolution. In particular, the previously accepted theories proposed that globular clusters were among the first bound structures formed in the universe, formed directly out of primordial material (e.g. Peebles \& Dicke 1968). This idea was particularly compelling because of the similarity between the predicted Jean's mass for primordial conditions, and the observed mass of globular clusters. The fact that globular clusters continue to form at the present time provides us with a window into the earlier universe when their formation was prevalent.

Not only did the Hubble Space Telescope spark the flurry of research in this area, but it has also been the work-horse; the vast majority of super star cluster studies have utilized Hubble in some way. $\dagger$ Because of Hubble there is a sample of globular clusters spanning almost every galaxy type, with a range of ages and metallicities. Large statistical samples of SSCs have been compiled, and their luminosity function (and presumably mass function) is well constrained (a power-law of -2) and roughly universal. The effective radii of many SSCs have been directly measured (typically $\sim 5 \mathrm{pc}$ ), and these radii in turn provide constraints on cluster dynamics and evolution. These are only a few of the many results.

The advances in this field due to Hubble have been truly remarkable. However, at the time of this writing, there are no missions in the foreseeable future that will have capabilities comparable to Hubble in the optical wavelength regime. Given this prognosis, it is clear that further progress in SSC research will depend critically on utilizing other tools. With new observational capabilities becoming available in the infrared to radio wavelength regimes, it is likely that the next decade of SSC research will be dominated by studying their earliest stages of evolution, which these long wavelengths are particularly well-suited to probing. For this reason, understanding the details of individual massive star formation in the Milky Way has become all the more important for extragalactic research.

$\dagger$ See e.g. the proceedings for "The Formation and Evolution of Massive Young Star Clusters", Lamers, Smith, \& Nota eds, 2003. 


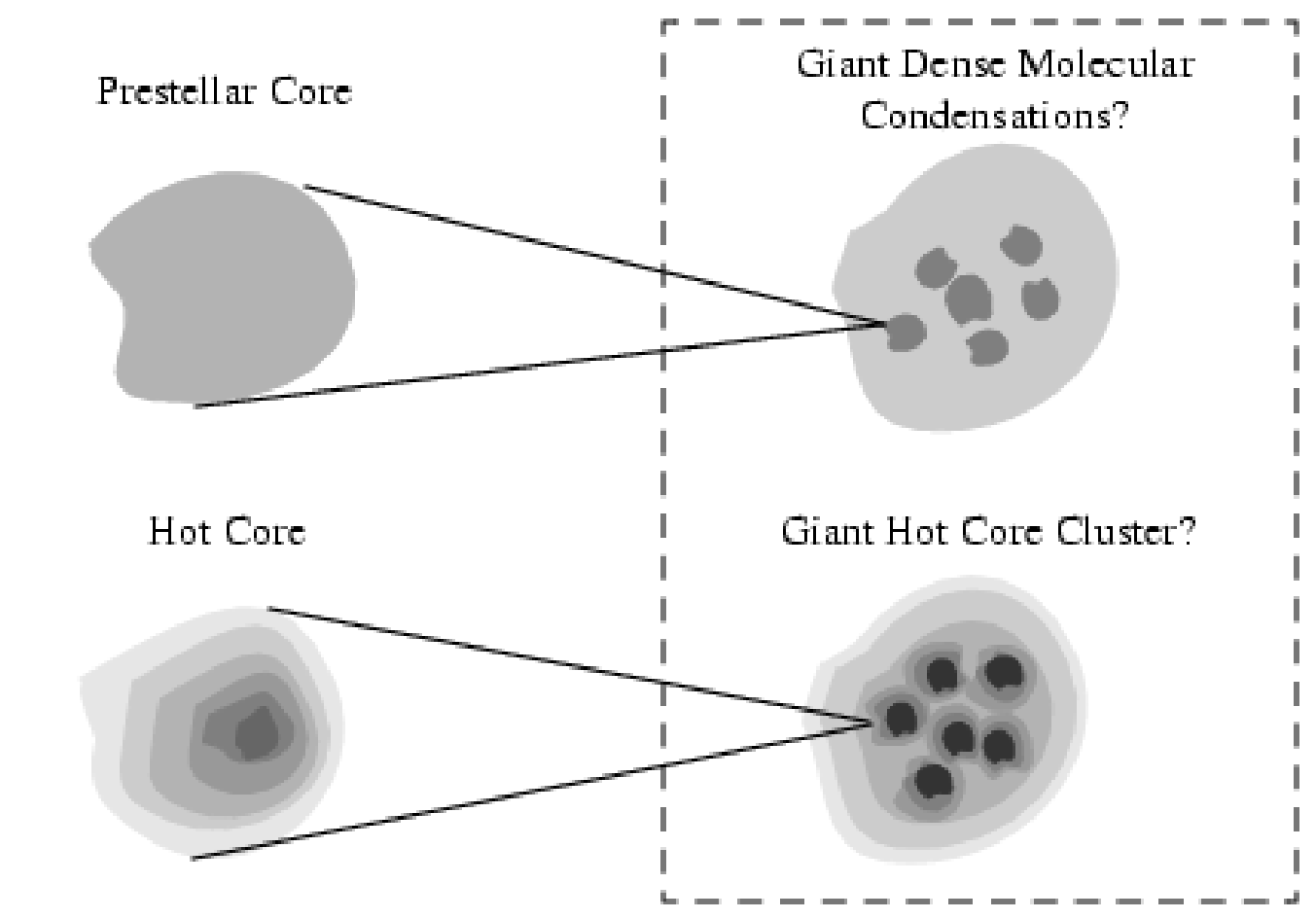

Ultracompact Hil Region

(UCHII)

Ultradense Hll Region

(UDH11)

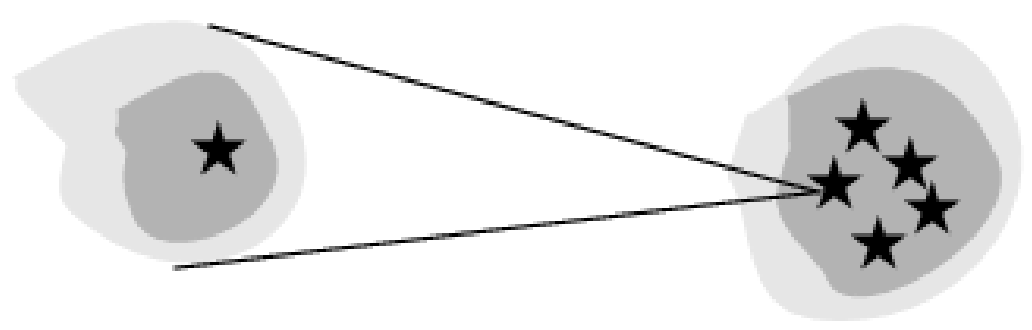

OB Star (with disc?)

Super Star Cluster

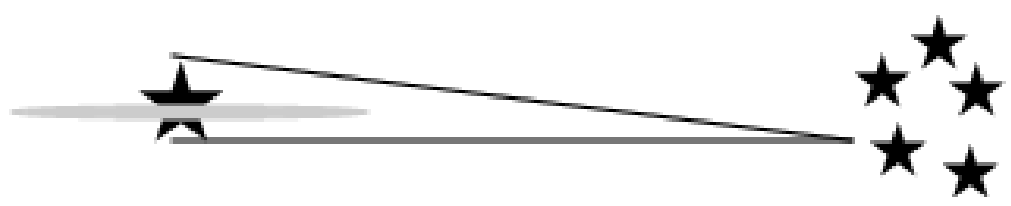

Figure 1. A cartoon illustrating a parallel evolutionary sequence for individual massive stars and super star clusters. The dashed box identifies the stages of super star cluster evolution that have not been observationally identified. 


\section{Connections to Galactic Star Forming Regions}

There is, of course, a rich history of studying star formation in our own Galaxy, as is highlighted in much of this volume. While there are clearly many important issues remaining in regard to massive star formation, a great deal has also been learned. Much of this knowledge can be applied to extragalactic objects, which cannot be observed with as much detail and precision as their galactic counterparts.

Observations of natal SSCs suggest that the early stages of massive star cluster evolution may parallel those of individual massive stars observed in the Milky Way. In particular, natal SSCs appear to have spectral energy distributions similar to ultra compact HII regions; these infant clusters typically have been identified at radio wavelengths as compact thermal (often optically thick) free-free sources, and they have copious amounts of thermal infrared emission. Like individual massive stars, SSCs also appear to spend $\sim 10-20 \%$ of the massive star lifetimes in a stage analogus to ultra compact HiI regions (Kobulnicky \& Johnson 1999, Vacca, Johnson, \& Conti 2002). These similarities suggest that we can apply many of the tools, techniques, and ideas regarding massive star formation in our own galaxy to objects that are much more distant. Figure 1 illustrates an analogy between the evolution of an individual massive star and a SSC.

While the analogy between individual massive stars and SSCs is a good starting point, the extent to which this analogy holds true will depend on a variety of factors. For example, if star formation within a SSC is not "instantaneous" to within $<1$ Myr, then the constituent stars in a cluster will not all be in the ultracompact HiI region stage simultaneously, which may result in different global SEDs. Extrapolating from what we know about individual massive stars to SSCs could also get us into trouble because of the extreme proto-stellar densities present in natal SSCs. It seems likely that we will gain the most physical insight not necessarily from the extreme cases alone, but also from trying to understand how the properties of star formation scale between individual massive star formation and SSC formation.

\section{What We Think We Know About Natal Super Star Clusters}

To date, the attempts to constrain the physical properties of natal clusters have been fairly crude, largely due to the limited observations available. As a result, we are forced by Achem's razor to the most simple solution, one which requires the least free parameters. Our current concept is the following: When an SSC is born, the massive stars ionize the surrounding natal material, creating an extremely dense HiI region, similar to a galactic ultracompact HII region, but scaled up in mass and luminosity. This dense HiI region is in turn surrounded by a dust cocoon that is presumably quite warm on the inner boundary. The constituent ionizing stars may be surrounded by individual Hil regions and dust cocoons in the earliest stages, but this is currently unknown. Clusters that are more sparse are likely to resemble ultracompact HiI region complexes in the Milky Way (such as W49A), and contain spatially discrete HII regions and cocoons, but the existence of individual cocoons becomes less tenable in the extreme stellar densities of the cores of massive SSCs.

These components of a natal cluster have separate observational signatures. The dense HII regions are characterized by an "inverted" radio spectral energy distributions due to optically thick free-free emission $\left(\alpha>0\right.$, where $\left.S_{\nu} \propto \nu^{\alpha}\right)$. The frequency at which the radio emission becomes optically thick is largely dependent on the density of the HII region. In the earliest stages of cluster evolution, most or all of the stellar luminosity is reprocessed by the surrounding dust cocoon, and this cocoon is observable at thermal 
infrared to sub-millimeter wavelengths. The nature of the resulting spectral energy distribution depends on whether the dust in the cocoon is smooth or clumpy (as will be discussed in $\S 6$, but this is not currently constrained by observations.

Existing observations have provided estimates for some of the physical properties of natal clusters, including size, electron density, pressure, stellar mass, HiI mass, dust mass, and age. For the most nearby clusters, their size can be directly measured using high spatial resolution observations. For more distant clusters, model HiI regions can be used to fit the radio spectral energy distribution and infer both the radius and electron density. The embedded SSCs that have been analyzed in this way (e.g. those in He 2-10, Johnson \& Kobulnicky 2003) have HiI region radii of only a few parsecs, and the electron densities have global values of $\sim 10^{3} \mathrm{~cm}^{-3}$, with peak values in excess of $\sim 10^{6} \mathrm{~cm}^{-3}$. Assuming temperatures of $\sim 10^{4} \mathrm{~K}$, these densities imply global pressures of $P / k_{B}>10^{7} \mathrm{~K} \mathrm{~cm}{ }^{-3}$ and peak pressures reaching values of $P / k_{B} \sim 10^{10} \mathrm{~K} \mathrm{~cm}^{-3}$.

The masses of the natal clusters, HiI regions, and dust cocoons can be inferred from radio and infrared observations. The optically-thin thermal flux (typically measured with the highest available radio frequency) can be used to infer a total ionizing luminosity, and the ionizing luminosity can be translated into an embedded stellar mass by assuming a stellar initial mass function. Using this technique, clusters with a range of masses have been detected (spanning modest OB-associations to massive SSCs). The HII mass can be estimated given the radii and densities for the ionized regions. In at least some cases, the HıI mass has anomalously low values $(<5 \%$ of the stellar mass, Johnson \& Kobulnicky 2003), which we tentatively interpret as a sign of the youth of the HiI regions. The mass of the dust cocoons around embedded SSCs has also been estimated in a few cases using infrared observations; these estimates have suggested dust masses of $\sim 10^{5}-10^{6} M_{\odot}$ for massive SSCs in SBS0335-052 and He 2-10 (Plante \& Sauvage 2002, Vacca, Johnson, \& Conti 2002).

\section{An Important Case Study: Henize 2-10}

A critical and unresolved issue in the study of massive star clusters is whether SSCs are just the statistical tail of a continuous mass distribution that extends all the way down to individual massive stars, or whether they can only be formed in special environments. While there is some evidence that supports each side of this debate, the answer has been clouded by observational and evolutionary issues. In typical optical studies, a cluster's age, extinction, and mass are all contributing factors to its observed luminosity and inferred mass. Moreover, as clusters evolve, the lower mass clusters will tend to be disrupted on time-scales as short as a few million years (Portegies Zwart et al. 2002), causing further confusion when interpreting cluster luminosity and mass functions. Radio observations of natal clusters are immune to reddening and sample only the youngest clusters before they have had time to evolve. As a result, only radio observations are capable of directly sampling the cluster mass function at the time of their birth.

The starburst galaxy Henize $2-10$ is one of the nearest galaxies (9 Mpc) known to host multiple natal SSCs detected in radio and infrared observations, as well as numerous SSCs that have already emerged to be visible in optical light (Kobulnicky \& Johnson 1999, Johnson et al. 2000, Vacca, Johnson, \& Conti 2002, Johnson \& Kobulnicky 2003, Cabanac, Vanzi, \& Sauvage 2005). These properties make Henize 2-10 a very important case study for examining the properties of natal SSCs. In our previous radio observations, four natal SSCs were detected, all with inferred masses in the range of $\sim 1-5 \times 10^{5} M_{\odot}$. Although the observations were sensitive enough to detect SSCs with somewhat lower masses, less massive natal clusters masses were not apparent. Given the linear resolution 

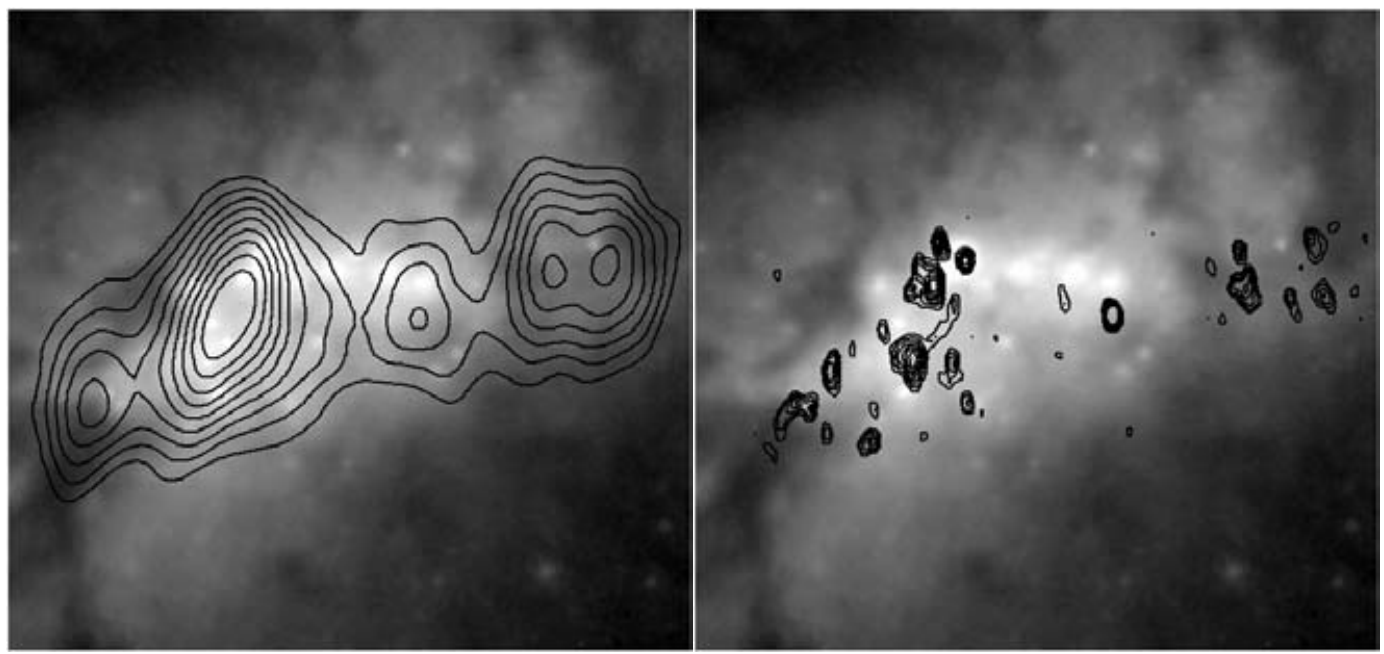

Figure 2. A comparison between the old (left) and new (right) radio observations from the Very Large Array (shown in contour). The gray-scale image in each panel is an I-band image from HST. The previous radio observations had a linear resolution of $\sim 20 \mathrm{pc}$ (Kobulnicky \& Johnson 1999, Johnson \& Kobulnicky 2003), while the new observations that include the Pie Town antenna have a linear resolution of $\sim 4$ pc (Biswas \& Johnson in prep). It is clear that the new radio observations will shed light on many of the issues related to SSC formation.

of the observations used for these previous radio studies of $\sim 20 \mathrm{pc}$, it seemed to be likely that some lower mass clusters were missing due to crowding and confusion.

In order to address the question of whether Henize 2-10 is only forming SSCs with masses of $\sim 10^{5} M_{\odot}$, or whether lower mass clusters are present, but had not yet been detected or disentangled from the more luminous sources, we obtained new high-resolution observations with the Very Large Array using the additional Pie Town antenna. A comparison between the old and new radio observations is shown in Figure 2 (Biswas \& Johnson in prep). In the new observations, it is clear that many of the previously known radio sources are composed of multiple components. Although these observations are also roughly an order of magnitude more sensitive than the previous observations, it is not clear that any additional natal SSCs exist outside of the main star forming region apparent in the previous radio observations. The analysis is still underway at the time of this writing, but it is clear that these new high-resolution and extremely sensitive radio observations will shed light on many issues related to SSC formation.

\section{The Infrared Properties of Natal SSCs: Results from 3-D Monte Carlo Radiation Transfer Models}

With the Spitzer Space Telescope providing a wealth of new observations, and Herschel, SOFIA, and ALMA on the horizon, appropriate physical models of natal SSCs are required in order to analyze data from these facilities. While the simple conceptual picture discussed in $\S 4$ is a good place to start, we know that the universe is complicated, and sometimes nice well-behaved uniform spheres just will not fit the data. One of the main difficulties that must be overcome in order to properly model star forming environments is that the ISM is not smooth, and therefore 1-D models cannot reproduce physical structures that are consistent with the observed ISM. We have produced a suite of models of natal SSCs using the 3-D Monte Carlo radiation transfer code described 


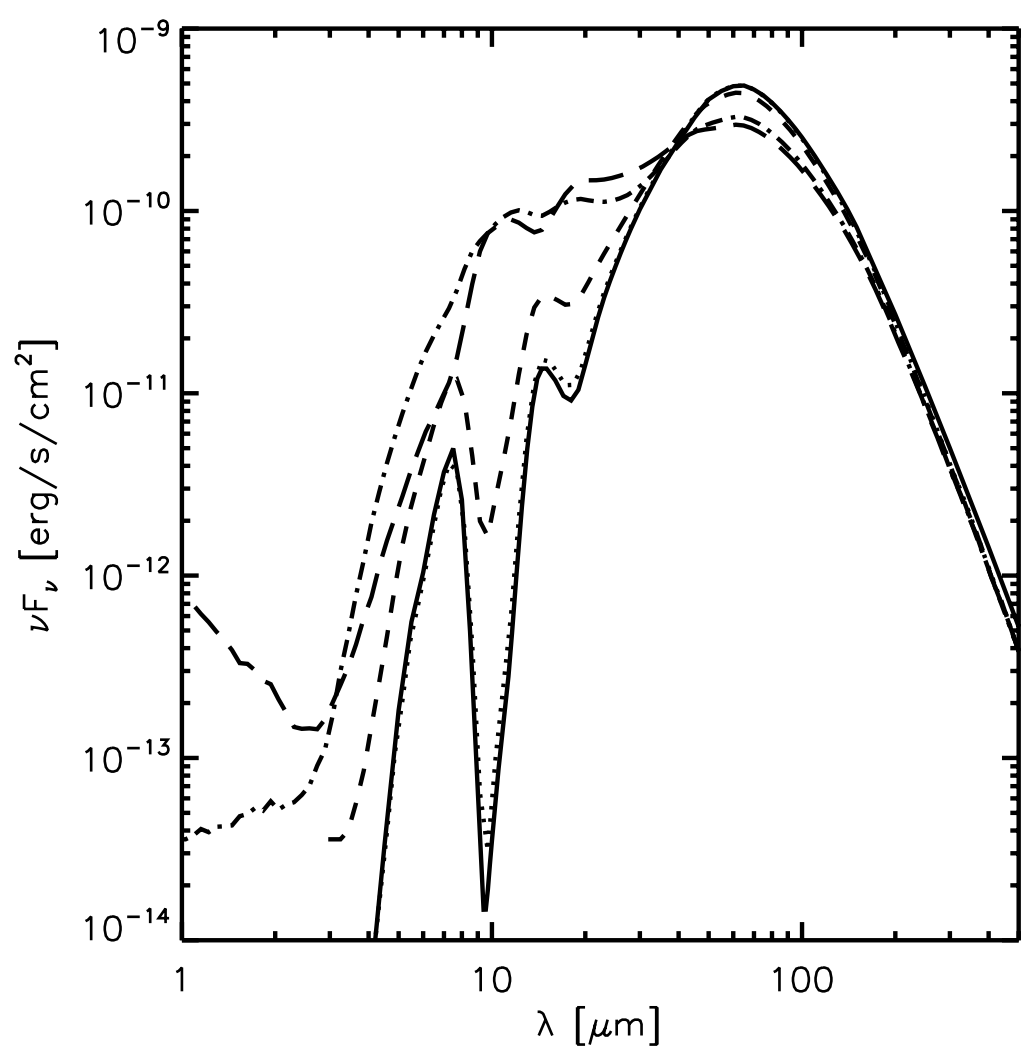

Figure 3. A comparison between the modeled spectral energy distribution for a natal SSC using different fractions of clumpy dust. The models shown are for a $10^{6} M_{\odot}$ stellar cluster formed with $10 \%$ star formation efficiency, an outer dust cocoon radius of $25 \mathrm{pc}$, and an inner cocoon radius of $1 \mathrm{pc}$. The solid line is $100 \%$ smooth dust, dotted line is $90 \%$ smooth, short-dashed is $50 \%$ smooth, long-dashed is $10 \%$ smooth, and dot-dashed is $1 \%$ smooth. Note that this figure is shown on a log-log scale, and the differences between the models can be quite significant.

by Whitney et al. (2003). These models allow for arbitrary density distributions, and we have invoked a clumpy fractal structure, consistent with observations of the ISM.

The differences in the spectral energy distribution (SED) between smooth and clumpy models can be very significant, as shown in Figure 3. One of the main differences is that clumpy dust allows more near-infrared light (both scattered and direct from the central source) to escape. More reprocessing of the photons also occurs in smooth dust cocoons, which changes the shape of the resulting SED. Spectral features, such as the silicate line at $\sim 10 \mu \mathrm{m}$ can also vary dramatically depending on the clumpy fraction for model with otherwise identical parameters (the silicate feature is much more strongly absorbed in models with a larger smooth fraction).

One important result is that the mid-infrared SED of the same object can vary significantly depending on the viewing angle if the dust cocoon is moderately clumpy. This is very bad news from an observational perspective; this degeneracy makes it impossible, regardless of the number of data points that sample a given SED, to determine a unique set of physical parameters that will fit the observations. The good news is that the 
degeneracy introduced by clumping is less significant at far-infrared wavelengths, although these wavelengths also suffer from poor spatial resolution.

These results should serve as a strong warning that cannot be overstated to investigators with thermal-infrared observations: Using 1-D models could lead to a significant misinterpretation of the data.

There is a vast amount of parameter space to explore, even without allowing for 3-D structure. The current study is limited to investigating the effects of a varying the inner and outer radius of the dust cocoon, total initial dust mass of the cocoon (which directly reflects the star formation efficiency), and the fraction of the dust that is clumpy. Within this limited parameter space, we have simulated an evolutionary sequence for SSCs as they go from deeply embedded to only having a thin dust shell. The results from this evolutionary sequence can be directly compared to observed colors of ultra compact HII regions.

Figure 4 shows a comparison between the colors of natal SSCs from our pseudoevolutionary sequence produced by our 3-D models and the observed IRAS colors of ultra compact Hir regions as well as "field" objects (Johnson et al. in prep, Kurtz, Churchwell, \& Wood 1994). Overall, there is excellent agreement between our model colors and the observed colors of ultra compact HII regions. In general, as a natal SSC moves through our pseudo-evolutionary sequence (its inner radius becomes larger and therefore the dust becomes cooler), its IRAS colors become more red. The most deeply embedded objects lie in the lower left region of the spread of model points, which reflects the extremely hot dust in the inner portions of the dust cocoons. If these objects with such hot dust exist, the lifetime of this state is likely to be extremely short. Therefore, embedded star forming regions with such hot dust should be quite rare, possibly accounting for the lack of ultra compact HiI regions detected with colors as blue as the models can produce. The model colors also extend further red-ward than observed for ultra compact HiI regions, but this is not surprising; as an embedded star forming region evolves and begins to emerge from its cocoon, its thermal-infrared luminosity will fall precipitously. As a result, we expect that the existing samples of ultra compact HiI regions become incomplete at these later stages (or perhaps objects that are this evolved are no longer considered to be ultra compact HiI regions!).

\section{Some Major Unresolved Questions}

There are no lack of questions regarding the birth of SSCs, and given the new observatories that are becoming available, it is an exceptionally good time to be asking these questions. A few major questions keep recurring that I want to highlight here:

(1) How does star formation vary between individual massive stars and SSCs (and is this reflected in the stellar initial mass function)?

(2) What are the environmental requirements necessary to form a SSC, and how do they differ from individual stars?

There are also a variety of properties that it would be nice to know more about. For example, it would be instructive to directly measure densities, pressures, and temperatures using various line diagnostics at IR to radio wavelengths. Another quantity that might be particularly useful for theorists (that is almost completely unconstrained) is the star formation efficiency in these objects. We also have no idea how much ionizing radiation could be escaping from the natal cocoons (and it could be quite a lot if the dust is clumpy). In fact, we know almost nothing about the natal dust cocoons (including the temperatures, grain compositions, and geometry). This list is certainly not exhaustive. An understanding of these quantities, and how they relate to the properties of the 


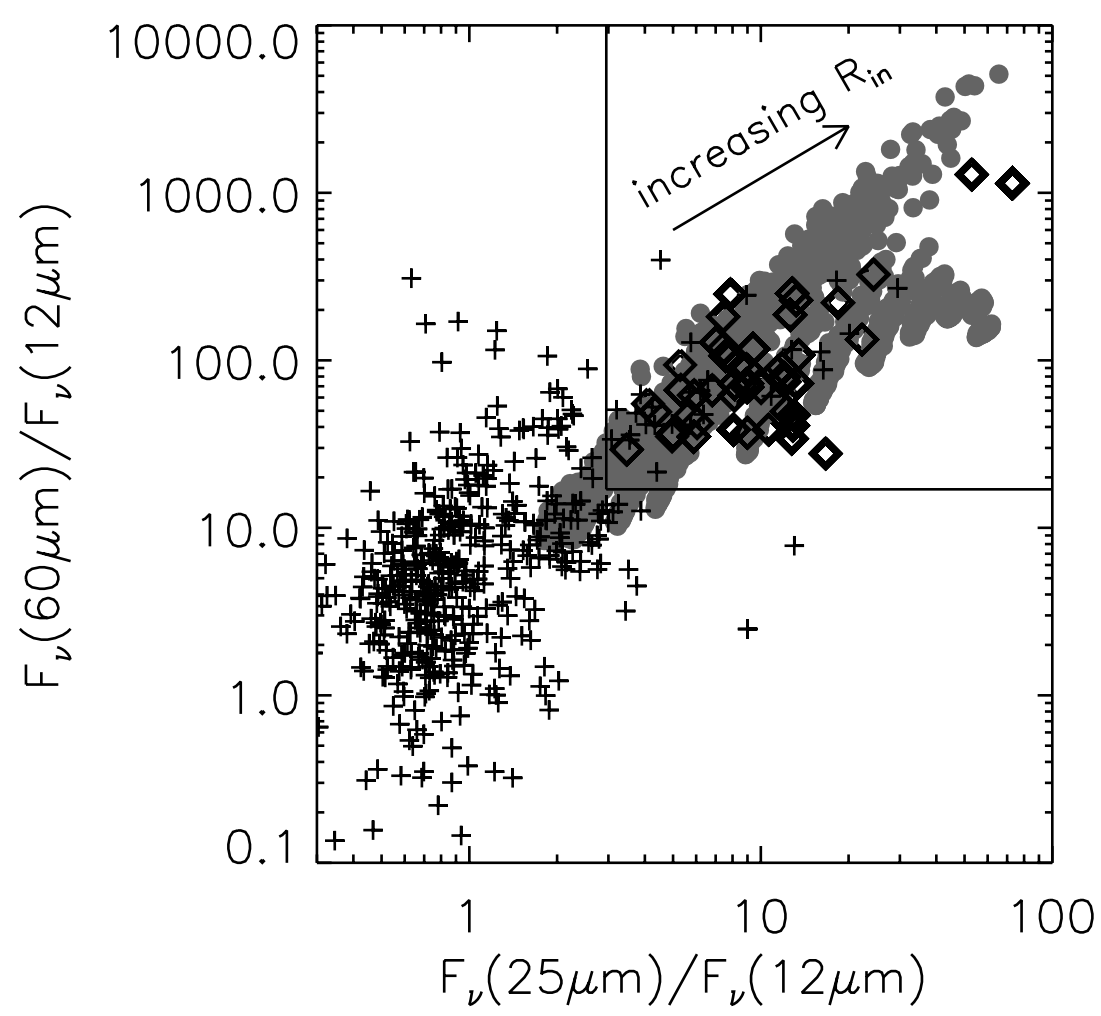

Figure 4. The IRAS colors from a subset of our models (gray circles) are compared to field objects (plus signs) and ultra compact HiI regions (diamonds) from the Kurtz, Churchwell, \& Wood (1994) survey. The models shown are for a $10^{6} M_{\odot}$ stellar cluster formed with $10 \%$ star formation efficiency, and an outer dust cocoon radius of $25 \mathrm{pc}$. The inner radius evolves through our pseudo-evolutionary sequence and moves outward from $0.1 \mathrm{pc}$ to $24 \mathrm{pc}$. All of the sight lines are shown, resulting in the scatter. The box shown in the upper right is the color criteria defined by Wood \& Churchwell (1989) to select ultra compact HiI regions.

resulting cluster, will eventually provide a great deal of insight into the requirements for massive star cluster formation.

\section{Looking Forward to the Future}

Over the next decade (or so), a number of powerful new facilities are going to become available at infrared to radio wavelengths. These observational capabilities are likely to bring about a renaissance in the study of the formation of massive clusters. These facilities will be incredibly well-suited to observing natal SSCs (Figure 5), and will allow us to study natal SSCs in amazing detail. Surely the new observations will bring about many new discoveries as well as many more new questions.

\section{Acknowledgements}

The work has benefited from many fruitful discussions with my collaborators, including I. Biswas, P. Conti, M. Goss, L. Hunt, R. Indebetouw, H. Kobulnicky, W. Vacca, B. Whitney, and K. Wood. I also gratefully acknowledge support for this work provided 


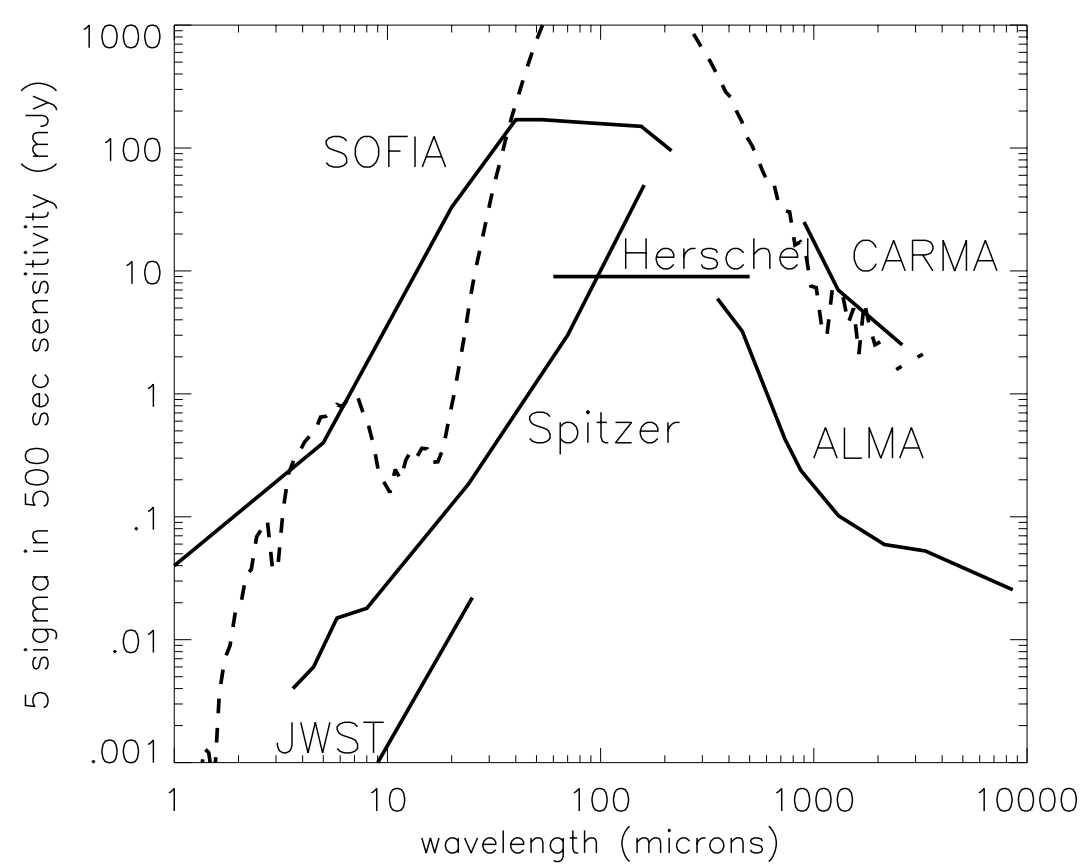

Figure 5. The anticipated sensitivities for upcoming infrared and millimeter observatories normalized to $500 \mathrm{sec}$ of integration time. A sample model spectral energy distribution for a $10^{6} M_{\odot}$ natal SSC at a distance of $10 \mathrm{Mpc}$ is over-plotted (dashed line) for comparison (Johnson et al. in prep). These new observing facilities are extremely well-suited to probing the birth of SSCs.

by the NSF through and Astronomy and Astrophysics Postdoctoral Fellowship, and by NASA through the Hubble Fellowship Grant \#01173.02-A through the Space Telescope Science Institute, which is operated by the Associated Universities for Research in Astronomy, Inc., under NASA contract NAS5-26555.

\section{References}

Biswas, I. \& Johnson, K.E. in prep.

Cabanac, R.A., Vanzi, L., \& Sauvage, M. 2005, ApJ, 631, in press

Fall, S.M. \& Zhang, Q. 2001, ApJ, 561, 751

Heckman, T.M. 2001, in: Gas and Galaxy Evolution, ASP Conference Proceedings, Vol. 240, p. 345

Kobulnicky, H.A. \& Johnson, K.E. 1999, ApJ, 527, 154

Kurtz, S., Churchwell, E., \& Wood, D.O.S. 1994, ApJS, 91, 659

Johnson, K.E., Whitney, B.A., Indebetouw, R., \& Wood, K. in prep.

Johnson, K.E. \& Kobulnicky, H.A. 2003, ApJ, 597, 923

Johnson, K.E., Leitherer, C., Vacca, W.D., \& Conti, P.S. 2000, AJ, 120, 1273

Peebles, P.J.E. \& Dicke, R.H. 1968,ApJ, 154, 891

Plante, S. \& Sauvage, M. 2002, AJ, 124, 1995

Portegies Zwart, S.F., Makino, J., McMillan, S.L.W., \& Hut, P. 2002, ApJ, 565, 265

Vacca, W.D., Johnson, K.E., \& Conti, P.S. 2002, AJ, 123, 772

Whitney, B.A., Wood, K., Bjorkman, J.E., \& Wolff, M.J. 2003, ApJ, 591, 1049

Wood \& Churchwell 1989, ApJ, 340, 265 